\title{
GLUE FOR DENTURE- DENTURE ADHESIVE-A REVIEW
}

Pratibha Katiyar ${ }^{1}$, Anupama Nigam ${ }^{2}$

\section{HOW TO CITE THIS ARTICLE:}

Pratibha Katiyar, Anupama Nigam "Glue for denture - denture Adhensive - A Review". Journal of Evolution of Medical and Dental Sciences 2013; Vol2, Issue 38, September 23; Page: 7373-7380.

ABSTRACT: Following the loss of natural dentition the edentulous individual can be faced with a number of problems associated with wearing complete dentures. One of the most common is that of looseness of either or both of the dentures. Dentures function as a substitute for missing teeth in mouth, while dentures are carefully fitted for the user; the fit can change over time, causing discomfort and slippage. To alleviate the discomfort and to control over the slippage, a denture adhesive may be applied to the denture. Successful complete denture therapy must involve both technical excellences during prosthesis fabrication and effective patient management. once the denture are placed, satisfying the expectations of many patients for optimal retention, stability of dentures is often beyond the technical skills of even the most accomplished practitioner. Discussing and implementing judicious use of denture adhesives may satisfy patient's expectation and also help to achieve the intended treatment goals. They indicated for routine use when appropriately constructed CD does not satisfy stability and retention expectations of patients.

KEYWORDS: Denture Adhesive, Denture Glue,

\section{GLUE FOR DENTURES - DENTURE ADHESIVE:}

Following the loss of natural dentition the edentulous individual can be faced with a number of problems associated with wearing complete dentures.

One of the most common is that of looseness of either or both of the dentures. Dentures function as a substitute for missing teeth in mouth, while dentures are carefully fitted for the user; the fit can change over time, causing discomfort and slippage.

To alleviate the discomfort and to control over the slippage, a denture adhesive may be applied to the denture.

More than 50 years ago it was suggested that local factors were primarily responsible for edentulous ridge resorption.

Schlosser implicated ill-fitting dentures and associated trauma to oral tissues as the primary causes of rapid destruction of the denture bearing structures. He lists faulty impressions, excessive occlusal vertical dimension, inaccurate centric jaw relationships, and occlusal disharmony as major contributing factors. Lammie suggested that a detrimental external molding force may adversely impact the residual bony ridges as overlying oral soft tissues contract or atrophy with time. This molding force may, in turn, accelerate resorption of the edentulous ridges.

The objective of complete denture therapy for patients with severe reduction of residual ridges is not solely the replacement of missing teeth. Rather, complete dentures must be designed to replace both the missing dentition and associated supporting structures.

Complete maxillary and mandibular dentures have long been considered the standard of care for treating edentulous patients. While most edentulous patients express relative satisfaction with their maxillary complete dentures, many do not enjoy equally successful mandibular denture comfort and function. 
The use of endosseous dental implants to assist in the support, stability, and retention of removable prostheses is now considered an effective treatment modality for the edentulous patient.

After thorough, evidence-based review of existing information at (McGill University), the following consensus statement was formulated: "The evidence currently available suggests that the restoration of the edentulous mandible with a conventional denture is no longer the most appropriate first choice prosthodontic treatment. There is now overwhelming evidence that a twoimplant overdenture should become the first choice of treatment for the edentulous mandible."

\section{RATIONALE:}

Successful complete denture therapy must involve both technical excellences during prosthesis fabrication and effective patient management. once the denture are placed ,satisfying the expectations of many patients for optimal retention, stability of dentures is often beyond the technical skills of even the most accomplished practitioner. Discussing and implementing judicious use of denture adhesives may satisfy patient expectation and also help to achieve the intended treatment goals.

They indicated for routine use when appropriately constructed CD does not satisfy stability and retention expectations of patients.

Some clinicians disapprove the use believing that they can be used with old dentures instead of having properly constructed accurately fitting denture Faulty, inappropriate use can lead to problems like impairment of the development of the NM system which is important in the retention and stability of the dentures.

\section{HISTORY:}

Denture adhesive has been introduced in modern dentistry in late 18th century.

The earliest patent pertaining to the adhesives was issued in 1913 and other patents followed this in the 1920's and 1930's. Till 19th century, they were used to hold base plates while recording jaw relation in immediate denture construction till final CD fabrication.

Types and composition of fixatives:

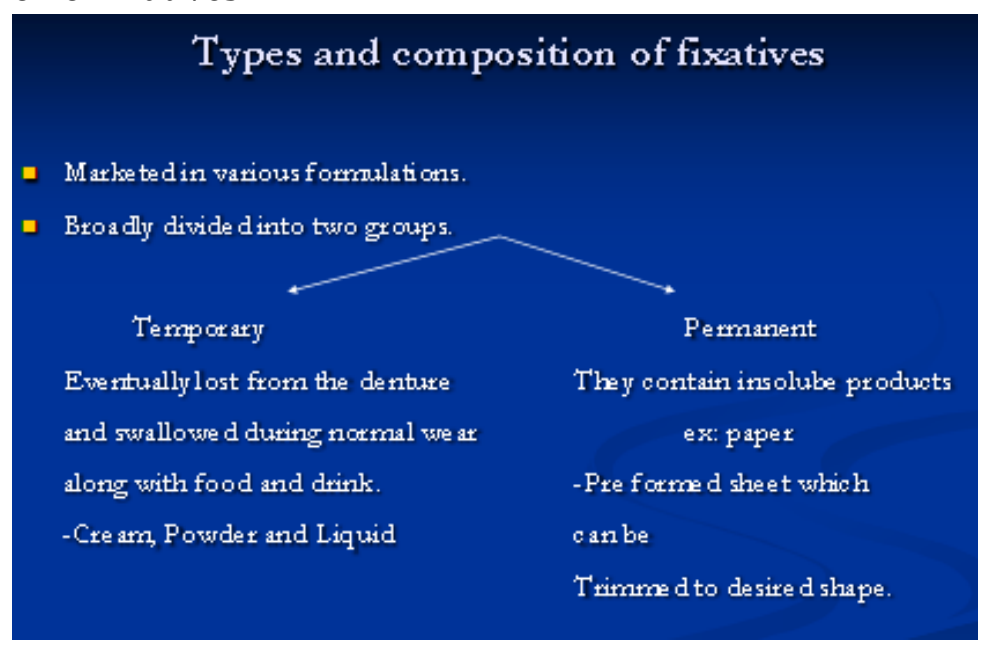

\section{COMPOSITION:}

All dentures adhesive have some basic ingredients 


\section{REVIEW ARTICLE}

- Adhesive components: ex: gelatin, pectin, Na carboxy methyl cellulose
○ $(10 \%-30 \% \mathrm{wt})$
$\circ$ These sensitize the adhesive to moisture, enhancing the cohesive property of formulation and enhancing gel strength.

- Antimicrobial agent: ex: Na borate, Hexachlorophene, or may be a combination of 8hydroxyquinoline or its salt, Nystatin, or Cu salts.(0.001\%-0.1\% wt)

- Action particularly against candida albicans

- Fillers: (dispersion agent):ex: Mg 02, NaPo4, CaSi

○ Prevent clumping of powder.

- Base: partially mixed salts of lower Alkyl Vinyl Ether/Malic acid, or Maleic Anhydride co polymers (GANTREZ) (10\%-50\%)

- Provides base or matrix for the adhesive material.

- $\quad$ Thickening agents: ex: petrolatum and mineral oil.(15\%-30\% wt)

$\circ$ To improve handling property.

- Flavoring agent: ex: peppermint oil, menthol.

- Preservative: ex: Benzoic acid, Na benzoate, parebens (0.03\%-0.6\% wt)

- Sweetening agent: ex: water proof artificial sweeteners (on formulation) (0.001-0.5\%)

- Colorant: ex: Tio2 dyes suitable for food drug and cosmetic application (FDC dyes) (indigo dye FDC-2) (0.001\%-0.5\%)

MODE OF ACTION: Effective of fixative depends on both physical and chemical forces and explained in terms of anionic /cationic interactions.

The adsorption of water and saliva by fixative results in formation of an anionic layer, that are attracted to cationic protein present in mucus membrane producing stickiness and form a barrier between denture and mucoperiosteum.

Carbonyl groups in the adhesive material provide strong bio adhesive and bio cohesive forces which improves the retention of the denture.

Adhesives comprise of water swell able gums/polymers suspended in oils and petrolatum, these hydrate and become tacky when introduced to saliva in oral cavity, thus holding denture in place.

According to ADA, it should have following characters:

- Product composition should be supplied

- Should not affect the integrity of the denture

- Biologically acceptable

$\circ$ Effective function as adherent

\section{Recommendations for use:}

Those who have Poor anatomical denture support though the denture are clinically and technically satisfactory.

Individuals with poor neuromuscular control like parkinsonism and stroke.

Xerostomia sufferer like Post radio therapy, drug induced, sjogren syndrome. 
To improve retention stability of the denture following insertion of new denture when difficulties are been experienced in developing NM control. (For limited period)

\section{Contra indications:}

1. For the retention of clinically unacceptable dentures, like
i. Worn out dentures,
ii. Relining cases,
iii. Pt's with physical inability to clean dentures.

2. Following insertion of immediate dentures to avoid post op discomfort.

3. Patients allergic to components of adhesives.

\section{Aspects of denture fixatives}

Clinical aspect: - Denture stability, retention and masticatory efficiency enhanced due to increased biting force with the use of denture fixative.

Minimal increase in VDO if not used in proper proportion.

Biological aspect: - Because of eventual loss from the denture along with the food and drink over a long period of time can lead to possible side effect like nausea, epigastric pain and allergic reactions (angioneurotic edema, hives)

Microbiological aspect: - Long term use of antimicrobial agent may influence the oral micro flora by selectively supporting the growth of some organism and inhibiting others.

Use should be limited in immune-compromised patients, as some can contain bacterial and fungal contaminants that may cause infections in these individuals.

\section{Advantages:}

1. Enhances the inter facial surface tensions between denture base and supporting tissues, improving the adhesive, cohesive and viscosity characteristics of interfacial film particularly in xerostomic patients.

2. Eliminating voids occurring in the interfacial space in the absence of absolute adaptation of the denture base to the bearing tissue.

3. Reduces the food impactions under the denture bases.

4. Improved chewing efficiency, increases bite forces, improve functional load distribution under the tissues.

5. Facilitate the psychological well being of the patient.

6. Patient with xerostomia:

a. Provides cushioning and lubricating effect, reduce frictional irritation, preventing the tissue dehydration.

7. Disadvantages

- Insoluble adhesives (synthetic wafers) cause tissue destruction, so better to use soluble adhesives.

- Support bacterial growth.

- Due to illiteracy of patient, improper use or over use of denture adhesives along with improper oral hygiene failure of adhesives is seen. 
It is appropriate to prescribe adhesive to augment retention and stability of conventional complete dentures. Anticipating suboptimal stability and retention in the presence of compromised patient factors, e.g., xerostomia and informing patients that the proper use of a limited amount of denture adhesive can supplement existing denture stability and retention. The need for denture adhesive is not necessarily an indication of suboptimal therapy, or admission of failure by either to dentist or patient.

Most denture wearers, at one time or another, have attempted to use adhesive to facilitate comfortable denture function. Unfortunately, the concept that "more is better" does not hold true for denture adhesives.

Appropriate Application of Denture Adhesive

1. Inform the patient that, due to existing conditions, achieving optimal complete denture retention and stability may not be possible. Also suggest that the proper use of denture adhesive is an acceptable means of augmenting the stability and retention of a new prosthesis. Honest and realistic communication of the anticipated results of therapy may ease future patient management problems.

2. The use of small amounts of hydrated paste adhesives (e.g., Fixodent, Procter \& Gamble) works well due to favorable adhesive, cohesive, and viscosity characteristics.
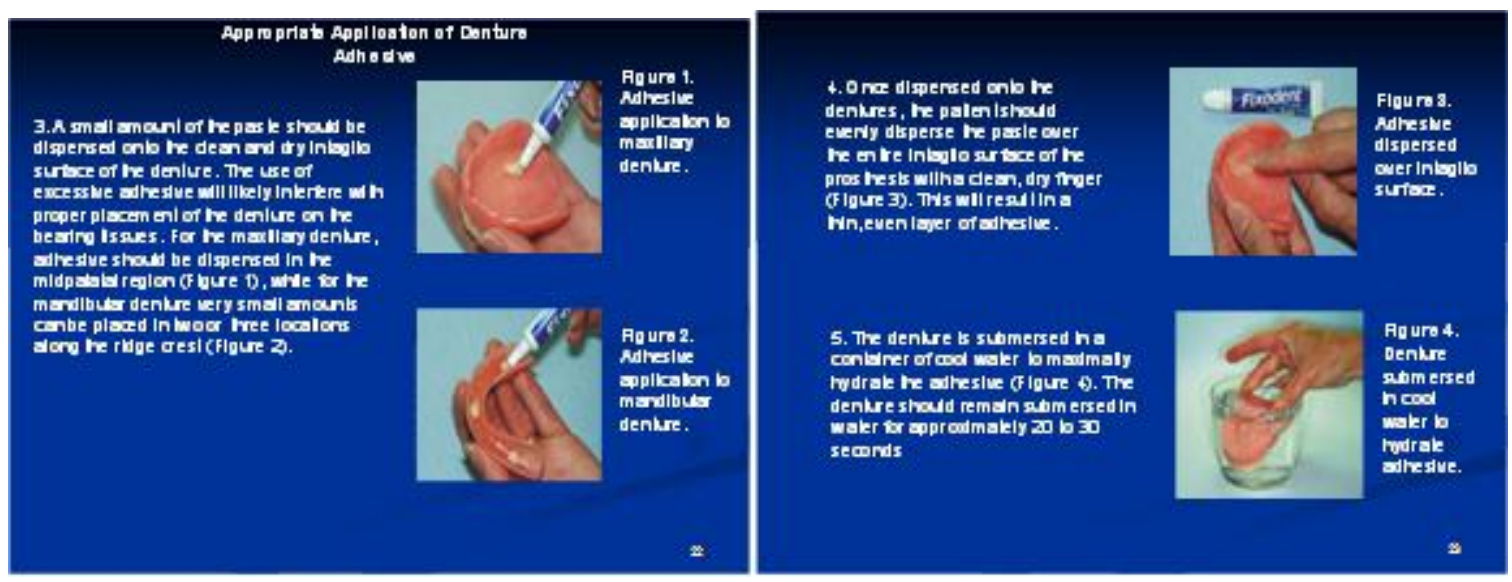


\section{REVIEW ARTICLE}

6. The denture is then placed in the mouth and immlyseated with inger pressure for approximately 10 seconds (Figure 5). Maintenance of seating pressure will cause the adhesive to flow throughout the inter tacial space between the denture base and the denture bearing sott tissues.

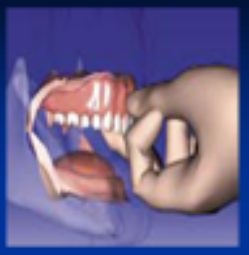

Figre 5.

Denture

placed

and imly

seated

7 . The patient maybe provided with the

sample container of adhesi ve used during

the demonstration. Suggesting local

stores that camythis product will

emphasize that adhesive use is a

component ofregular denture use. The

patient should be told that the use of

excessive adhesive may indicate an

inadequate it, necessitating denture

reline or remake procedures.

Techrique for Derture Adhesive Removal

1. Use of an electric toothbrush can enthance thorough cleaning of both denture surfaces and denture besring oral tissues (Figure 6). Inexpensive,

battery powered brushes are now widely

available to consumers (e.g., Crest

SpinBrush Pro. Procter $\&$ G amble). A

smallamount of toothpaste on the electric

toothbrust will serve to fresten the

patient's breath and improvedtaste

(Figure 7 ).

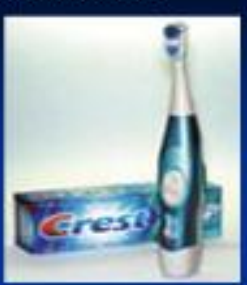

Figure 6 .

Battery

powered

toothbrush

and tooth

paste.

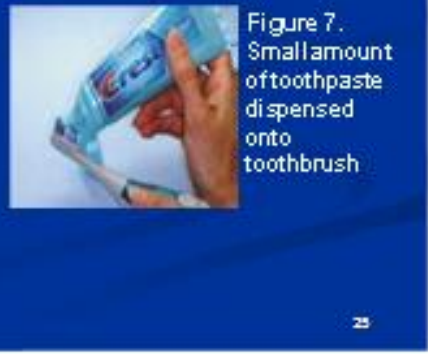

2. Rem ove the dentures from the m outh, and thoroughl y scrub the entire Intagllo zur tace of the dent ures with the electric toothbrush (F/gure B). Thls (intended to ellm Inate adhest ve from the dentures. $R$ ather, this al scrubbing will loosen residusi adhegl ve material, fadiltating

subsequent removal.

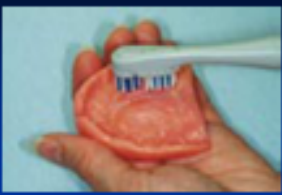

scrubblng of

the denture. 


\section{REVIEW ARTICLE}

3. Tle de iture b tie I le al sibmerged is a citalie ror warm water aid s in i the

ousy scribbed is lig tie e lectic tootibrisi (Figire 9). Fim pressi re sloikd be applled to the brisi II orde i be ilm Irate adies we from the de it re sirtece. Parteces or

clım ps of adies we $m$ aterla iw IIIbe see I is-

lig b tie si riace of tie wate i (Fig I re 10).

TI is proced i re is coitin ied in til the e i tire

de it re s i riace is free of res kital adieswe.
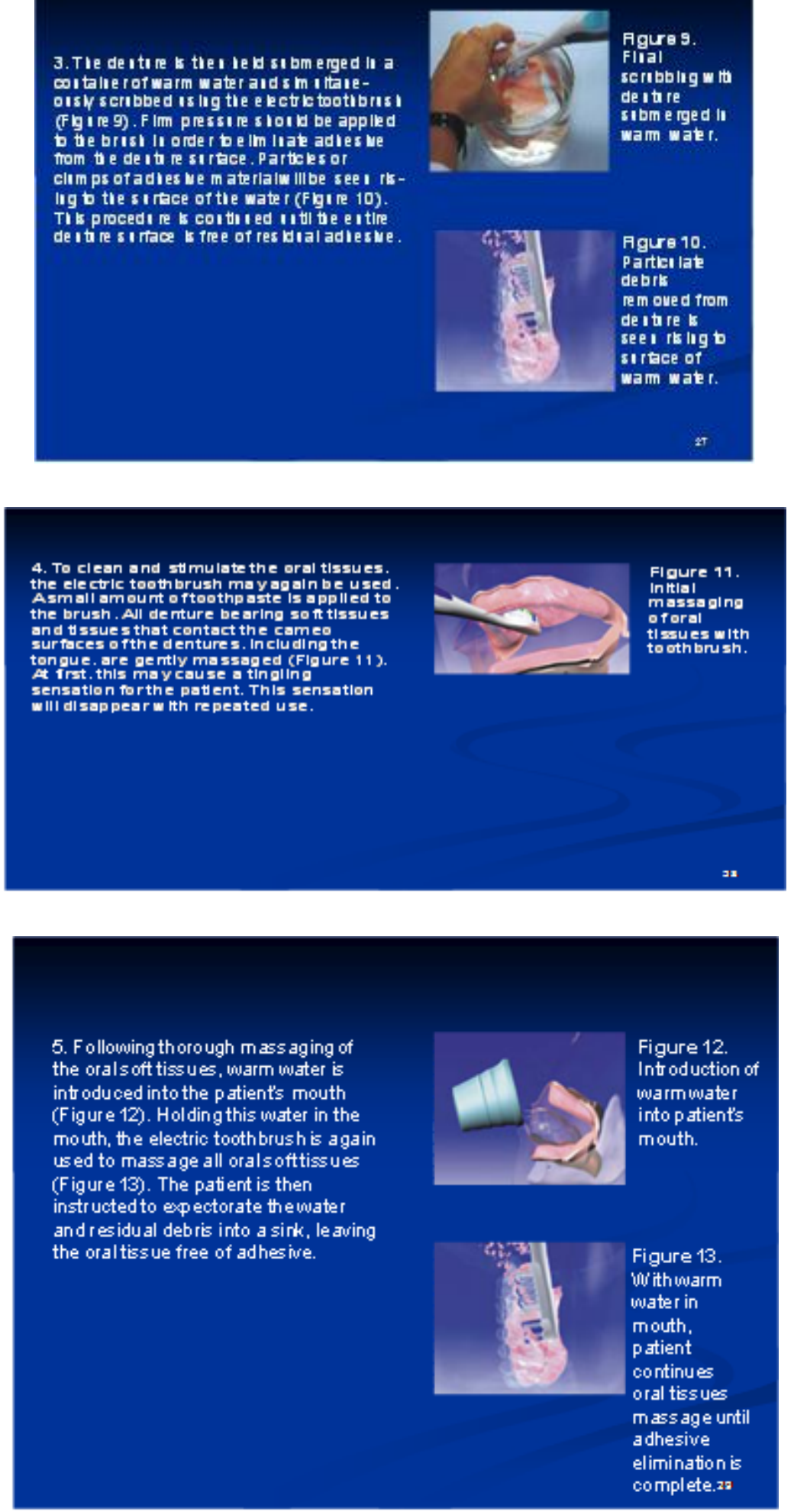

\section{REFERENCES:}

1. Dental resource net by crest.

2. Solving problems in Complete Dentures. 


\section{REVIEW ARTICLE}

3. J oral rehabilitation 2004, 31; 131-134.

4. Adhesives formulation, United States patent, Google search.

5. Essentials of CD prosthodontics, Winkler.

\section{AUTHORS:}

1. Pratibha Katiyar

2. Anupama Nigam

\section{PARTICULARS OF CONTRIBUTORS:}

1. Senior Lecturer, Department of Prosthodontics, Chandra Dental College and Hospital, Safedabad, Barabanki, Lucknow.

2. Reader, Department of Prosthodontics, Chandra Dental College and Hospital, Safedabad, Barabanki, Lucknow.
NAME ADDRESS EMAIL ID OF THE CORRESPONDING AUTHOR:

Dr. Pratibha Katiyar, 3/242, Vinay Khand 3 Gomti Nagar, Lucknow, U.P.

Email-dr_pratibha2002@yahoo.com

Date of Submission: 24/08/2013.

Date of Peer Review: 26/08/2013.

Date of Acceptance: 14/09/2013.

Date of Publishing: 23/09/2013 